\title{
EP-80
}

\section{First successful report of liver transplantation from donation after circulatory death}

\author{
Incheon KANG ${ }^{1}$, Jae Geun LEE ${ }^{* 2}$ \\ 'Department of Surgery, CHA Bundang Medical Center, CHA University School of Medicine, Korea \\ ${ }^{2}$ Department of Transplantation Surgery, Yonsei University College of Medicine, Korea
}

Introduction: In Korea, deceased donor liver transplantations (DDLT) have been performed using donation after brain death (DBD) donors, donation after circulatory death (DCD) has not been reported.

Methods: Despite five consecutive EEG tests, a 52-years old man who was not diagnosed with brain death was finally determined to donate an organ in DCD category III (awaiting cardiac arrest). The final donor organs were decided with liver and kidney, a 56-years old man with a Meld score of 27 was selected as a recipient of the liver.

Results: After the withdrawal of life-sustaining therapy, which includes termination of cardiopulmonary support and all essential medications, functional (true) warm ischemic time was ( $\mathrm{SBP}<50 \mathrm{mmHg}$ or $\mathrm{SpO}_{2}<70 \%$-asystole) 14 minutes and total warm ischemic time was 21 minutes. The liver was retrieved with the super-rapid technique, LT of the recipient was successfully performed. The recipient's recovery was uneventful and discharged on a postoperative day 37.

Conclusions: With an increasing demand for donor organs, DCD could be an alternative option to contribute to donor numbers. 